\title{
Rationalität - Wissenschaft - Technik
}

\author{
STEFAN BECK
}

Wie sich Denk- und Handlungsweisen anderer Menschen verstehen und repräsentieren lassen, ist eine der zentralen ethnologischen Fragen, die die Disziplin seit ihren Anfängen umtreibt. In ihrer Gründungsphase waren die unterschiedlichen Antwortversuche dabei durch einen »kognitiven Evolutionismus" geprägt, die Vorstellung, dass sich menschliches Handeln und Denken langsam aus irrationalen und durch magisches Denken geprägten Zuständen in einen aufgeklärten, zivilisierten Modus hinaufarbeitete, der durch Rationalität und ein wissenschaftliches Weltbild geprägt sei. Das Studium vermeintlich »einfacher" Gesellschaften oder der nichtbürgerlichen Schichten in der Moderne schien für viele Ethnologen daher gleichsam einen Blick in die kognitive Kinderstube der Menschheit zu ermöglichen - in »Vorstufen" menschlichen Denkens. Für die Sozial- und Kulturanthropologie wurde diese Sicht ab den 1920er Jahren vor allem durch die Forschungen Bronislaw Malinowskis überwunden, in der Europäischen Ethnologie in den 1960er Jahren durch die Verabschiedung einer romantisierenden Perspektive auf das »Volksleben" - mit wichtigen Folgen für Erklärungs- und Darstellungsweisen des Faches. Die außereuropäische Ethnologie diskutierte die Frage der Rationalität und der Befähigung für wissenschaftliches Denken am Beispiel des Verhältnisses von Magie, Religion und Wissenschaft, die Europäische Ethnologie an der Frage, wie überwiegend »traditional« orientierte Lebensweisen durch den Einsatz moderner Technologien transformiert wurden.

Für die Perspektive der Europäischen Ethnologie sind diese veränderten Sichtweisen vor allem deshalb zentral, weil in diesen Debatten weniger 
danach gefragt wurde, was Rationalität sei, sondern wann Menschen in welchen Zusammenhängen auf unterschiedliche Arten einer - mehr oder weniger - rationalen Erklärung von Phänomenen zurückgriffen. Die Fragen jedenfalls, wann Akteure auf "rationale Erklärungsmodelle" zurückgreifen, wie sie Handlungen und Ereignisse interpretieren, welche Denkstile dabei leitend sind - diese Fragen erweisen sich bei der Analyse von Praktiken, in denen Wissenschaft und Technik eine zentrale Rolle spielen, als überaus fruchtbar.

\section{Weiterführende Literatur}

Tambiah, Stanley Jeyaraja (1990): Magic, Science, Religion, and the Scope of Rationality, Cambridge: Cambridge University Press.

In seinem grundlegenden Überblick der Diskussionen der britischen und amerikanischen Anthropology zur Frage des Verhältnisses von Magie, Religion und Wissenschaft gelingt es Stanley J. Tambiah, die großen Linien des anthropologischen Paradigmen-Wechsels vom Lehnstuhl-Primitivismus des 19. Jahrhunderts hin zu einer sozialtheoretisch und empirisch argumentierenden Disziplin nachzuzeichnen. 


\section{Einleitung}

Die Philosophen hätten - so der kanadische Wissenschaftsforscher Ian Hacking - aus der Wissenschaft eine Mumie gemacht, aber als »sie endlich den Leichnam auswickelten und die Überbleibsel eines historischen Werdens und Entdeckens erblickten, führten sie zu eigenem Behufe eine Rationalitätskrise herbei. Das geschah um das Jahr 1960. Eine Krise war es, weil dadurch unsere althergebrachte Denkweise erschüttert wurde, nach der die wissenschaftliche Erkenntnis als krönende Errungenschaft der menschlichen Vernunft gilt.« (Hacking 1996: 13) Was die Wissenschaft zu einer Mumie machte, ist laut Hacking die Annahme eines kumulativen menschlichen Erkenntnisforschrittes, der sich durch den immer mehr verfeinerten Gebrauch der Vernunft (= Wissenschaft) eingestellt habe - eine Position, wie sie (bei allen Unterschieden) von Wissenschaftsphilosophen wie Carnap oder Popper vertreten wurde und die die Physik als Muster dieser Rationalität ansah. (WissenschaftstheORIE) Dafür, dass sich der Zweifel an dieser Annahme schließlich durchsetzen konnte, macht Hacking die Wissenschaftsgeschichte verantwortlich, die gezeigt habe, dass Wissenschaft weder kumulativ sei noch mit präzise definierten Begriffen arbeite und auch keine klare Trennung zwischen Theorien und Beobachtungen bestünde - Thesen, wie sie vor allem Thomas Kuhns Buch »Die Struktur wissenschaftlicher Revolutionen« popularisierte. (Kuhn 1976) Hacking zieht aus den in Kuhns Buch kulminierenden Debatten einen überraschenden Schluss: Seine Position ist, dass Rationalität in der Wissenschaft von geringer Bedeutung sei - und auch analytisch ermögliche nicht das Wort »rational«, sondern eher Worte wie »irrational«, »gescheit«, "phantasievoll« etc. interessante Einblicke in die Arbeitsweisen von Wissenschaftlern.

Hackings Thesen sind provokant - für die etablierte Wissenschaftsphilosophie ebenso wie für die Wissenschaftssoziologie; Sozial- und Kulturanthropologen hingegen können sie nicht recht überraschen. Allerdings sind die Gründe dieser Abgeklärtheit gegenüber der Wissenschaft anders gelagert: Während Hacking Wissenschaft durch ihre Historisierung entmumifiziert und vom Sockel gehoben sieht, erreichen vergleichend arbeitende Ethnologen dies durch die Sozial- und Kulturanthropologisierung menschlicher Rationalität. Für sie ist wissenschaftliche Rationalität in ihrer westlich-modernen Form nicht die höchste, sondern nur eine - allerdings 
machtvolle - Form unter anderen. In der internationalen Ethnologie setzte sich diese Einsicht zudem deutlich früher als in der Philosophie oder Soziologie durch - spätestens 1925, als Bronislaw Malinowski einen Essay unter dem Titel »Magic, Science and Religion« veröffentlichte (Malinowski 1948) und damit auch die modernistische Unterscheidung zwischen uns, die wir Rationalität praktizieren, und den Anderen, die dies (noch nicht) tun, fundamental in Frage stellte.

Im Folgenden wird die Frage nach der Rationalität wissenschaftlichen Handelns und des rationalen Umgangs mit Technologien aus dieser anthropologisch informierten Perspektive aufgegriffen. Charakteristisch hierfür ist, dass anders als in der Wissenschaftsphilosophie oder der frühen Wissenschaftssoziologie nicht versucht wird, eine »reine«, ort- und zeitlose Form der Erkenntnis oder rationalen Handels zu rekonstruieren, sondern stets vorausgesetzt ist, dass »rational« ein relativer Begriff ist - relativ in Bezug auf einen sozialen und materiellen Kontext, relativ zu einer umgebenden Kultur und relativ in Bezug auf die Gemengelagen, in denen sich behaupten lässt, Rationales zu finden und in denen sich Rationales behaupten muss; ${ }^{1}$ und schließlich relativ im Sinne von »Schattierungen « und »Abstufungen«. Aber auch hier gilt: analytisch taugt eher die Beobachtung von vermeintlicher Irrationalität, um Überraschendes herausfinden zu können. Hierzu möchte ich in einem ersten Schritt verdeutlichen, wie Feldforschung solche überraschenden Einblicke ermöglicht, dann zweitens vorstellen, wie in neueren Studien der Science and Technology Studies die Frage »rationalen Handelns« im Labor thematisiert wird. In einem dritten Schritt sollen knapp einige Anknüpfungspunkte zur Debatte um Magie, Religion und Wissenschaft vorgestellt werden, wie sie in der Sozial- und Kulturanthropologie beziehungsweise in der Europäischen Ethnologie erarbeitet wurden.

1 Ian Hacking (1983: 6) prägte für diese ort- und zeitlosen Formen der wissenschaftlichen Erkenntnis, wie sie in der Wissenschaftsphilosophie in der Tradition Carnaps oder Poppers rekonstruiert wurden, den Begriff der »flat formalities«. (WISSENSCHAFTSTHEORIE) Im Gegensatz dazu stellt sich die ethnologische Wissenschaftsforschung die Aufgabe, gleichsam »thick formalities « - also intensiv in Raum- und Zeitkontexte eingebettete Verfahrensweisen - zu rekonstruieren, wie sie bei der Produktion wissenschaftlicher Erkenntnisse, verstanden als Praxis, wirksam sind. (SOCIOLOGY OF SCIENTIFIC KNOWLEDGE) 


\section{SCHWARZE SÄULEN}

Ich möchte mit einem Auszug aus meinem Feldforschungstagebuch beginnen, das ich anlässlich einer Forschungsreise 2005 in die Türkei geführt habe. Im konkreten Fall geht es um eine Besichtigung einer reproduktionsmedizinischen Abteilung eines gerade eröffneten privaten Krankenhauses, des Anadolou Health Centers, das von der gleichnamigen, größten Stiftung der Türkei finanziert und in Kooperation mit der Johns-Hopkins Medical School betrieben wird. Die von mir besuchte reproduktionsmedizinische Abteilung arbeitet eng mit ihrer Schwesterabteilung in Baltimore zusammen, etwa indem Personal aus der Türkei in die USA zur Weiterbildung geschickt wird oder der Erfolg von Behandlungsprotokollen zwischen beiden Einrichtungen abgeglichen und bei Bedarf die jeweiligen Praktiken vor Ort modifiziert werden. Ich sprach mit dem leitenden Reproduktionsmediziner, Aytuğ Kolankaja, der seine gut gehende Privatpraxis einige Monate vor dem Besuch aufgegeben hatte, um die Leitungsposition in der Klinik zu übernehmen. Dabei nahm er offenbar zahlreiche seiner früheren Mitar-beiterinnen und Mitarbeiter mit. Als Motivation für diesen Wechsel nannte er, dass er nun Zugang zur führenden internationalen Forschung habe; dies sei viel wichtiger als viel Geld zu verdienen - er ließ durchblicken, dass er nicht mehr verdiene, als vorher. Ob dies zutrifft, konnte ich nicht beurteilen. Festgehalten habe ich im Feldforschungstagebuch folgende Beobachtungen, die während seiner Führung durch die Labors entstanden:

[...] Das Labor ist offenbar technisch auf dem neuesten Stand der Dinge. Bei der Führung durchs Labor hatte ich mich über merkwürdige, zwei Meter hohe schwarze Säulen gewundert, die in allen Laborräumen standen und deren Funktion mir nicht offenbar war. Ich fragte, um was es sich dabei handele und Aytuğ erklärte mit einer Haltung, die ich als Ausdruck von Stolz deutete, dass es sich dabei um ein neuartiges Filtersystem handele, das Feinstäube und Gas-Ionen aus der Laborluft entferne. Damit sei - so Aytuğ - eine bedeutende Erfolgsverbesserung bei der Implantation der Eizellen zu erzielen. Da ich bislang noch nie davon gehört hatte, dass für die Manipulation von Gameten Reinsträume notwendig seien (in den anderen Kliniken wurde allenfalls Operationsraum-Hygiene für notwendig erachtet), fragte ich nochmals nach dem Wirkmechanismus. Ich bekam jedoch keine klare Aussage, nur den Hinweis darauf, dass Gas-Ionen und Feinstäube offenbar von den Nährmedien auf- 
genommen würden, in denen die Eizellen und Embryonen manipuliert und aufbewahrt würden, von wo sie dann in die Zellen diffundieren könnten - dies sei allerdings bislang ein noch hypothetisches Modell. Jedoch seien - so Aytuğ - durch den Einsatz der Filter an einer US Forschungsuniversität sehr positive Ergebnisse erzielt worden. Deshalb hätte er nun beschlossen, verkleinerte Versionen auch in die »breeder« einzubauen, in denen die befruchteten Eizellen heranreifen.

Aytuğ nutzt den Hinweis auf diese Filter, deren Wirkweise wissenschaftlich noch gar nicht wirklich belegt ist, auch gegenüber den Patienten, um die Qualität seines Labors zu demonstrieren. Interessanterweise ist es dabei gerade der Hinweis auf die bislang nicht vollständig geklärte Wirkweise der Geräte, mit dem er begründet, dass die Patienten hier wirklich »the latest technology« bekämen, etwa in dem Sinn: Selbst wenn noch nicht geklärt sei, wie die Filter wirkten, werde nichts unversucht gelassen, um die Erfolgsquoten der Behandlung zu erhöhen. Mir ist nicht klar, ob die Säulen überhaupt an die Steckdose angeschlossen waren - es gab jedenfalls keine Betriebsanzeigen, Displays, Leuchtdioden oder ähnliches, die darauf hingewiesen hätten, dass sie eingeschaltet waren.

Schon bei der Führung hatte mich diese Aussage verwirrt - wieso investiert ein Wissenschaftler, der mir zuvor einen Vortrag über die zentrale Bedeutung evidenzbasierter Verfahren in der Medizin und die auf Dauer gestellte Evaluation der in der Klinik angewandten Behandlungsmethoden gehalten hatte, viel Geld in eine Technologie, deren Wirkmechanismus völlig ungeklärt ist, deren »Erfolg« (über die erwähnte, anekdotische Evidenz hinaus) bislang noch nicht belegt wurde und deren Einsatz - gemessen an rationalen Kriterien - zumindest fraglich ist? Handelt es sich hier um einen reinen »Werbegag«, der für die »Kunden« und Neugierigen wie unser Forschungsprojekt inszeniert wird? Oder geht es hier um Mehr? Handelt es sich hier um eine hochtechnisierte Form esoterischer Praktiken oder wirklich um »cutting-edge science«? Was »bewirken« die schwarzen Säulen im Labor tatsächlich - für Patienten, Beschäftigte, Eizellen und die Gas-Ionen?

Tatsächlich bin ich in Gesprächen mit Biologen oder Medizinern öfter an einen ähnlichen Punkt gekommen: Gerade in Forschungsfeldern, die neu sind, oder wenn Technologien angewandt werden, die noch nicht vollständig in Routinen oder standardisierte Verfahren eingebaut sind, wenn biologische Mechanismen, mit denen gearbeitet wird, noch nicht vollständig verstanden sind, wenn also Objektivierungspraktiken, die idealtypisches wissenschaftliches Handeln ja auszeichnen, an ihre Grenze kommen, dann 
häufen sich Redefiguren wie: »Sie hat ein besonderes Händchen« um Resultate zu erzielen, die anderen Labormitgliedern eben nicht gelingen wollen. Oder »so klappt es ganz gut«, das »hat sich bewährt«; oder auch »heute hat gar nichts geklappt«, es war »ein schlechter Tag, das muss am Wetter (oder an X, wobei X ein beliebiger externalisierter Faktor sein kann) liegen«. Solche Aussagen werden oft mit einem unsicheren Lächeln oder einem leisen, selbstironischen Unterton gemacht - halb entschuldigend, weil keine »harten«, wissenschaftlichen Begründungen gegeben werden können. Das Lächeln oder die Ironie hat in Gesprächen dann meist die Wirkung, dass weitere Nachfragen unterbleiben - man kam an eine Grenze des Sagbaren, die auch vom Gesprächspartner akzeptiert und weggelächelt wird.

Ich möchte im folgenden die hier sichtbar werdende Unsicherheit als einen Hinweis auf ein grundlegendes, in der Ethnologie und Anthropologie seit mehr als 100 Jahren intensiv diskutiertes Problem interpretieren: Auf die Frage nach der Rationalität des Handelns, die wir uns selbst und unseren Forschungspartnern gewöhnlich zubilligen, die wir fest in unsere Selbstbilder eingebaut haben - wobei alle Beteiligten möglicherweise wissen oder zumindest ahnen, dass es sich hier um eine freundliche Fiktion handelt. Und es wird um die Frage gehen, wie in der Moderne unterschiedliche Wissenstypen voneinander abgegrenzt werden, etwa wissenschaftliches von nicht-wissenschaftlichem Wissen; und darum, ob es sich hierbei um klare Gegensätze oder eher um Kontinuitäten handelt. Schließlich wird es um die ganz grundsätzliche Frage gehen, ob rationales Handeln als ein psychisch-kognitives (wie üblicherweise von der Philosophie vorausgesetzt) oder als ein sozial-kognitives (also: als anthropologisches) Phänomen anzusehen ist, bei dem materielle ebenso wie soziale Faktoren zentral sind.

\section{RATIONALITÄT UND MAgIE IM LABOR}

Die beiden kanadischen Wissenschaftsforscher Alberto Cambrosio und Peter Keating thematisieren in einem wegweisendem Aufsatz aus dem Jahr 1988 diese Problematik am Beispiel eines damals neuartigen biotechnologischen Verfahrens, das inzwischen zu einer der wichtigsten Techniken der biowissenschaftlichen Laborforschung wurde - der Hybridoma Technologie. Um was es sich dabei genau handelt, braucht hier nicht weiter zu interessieren - wichtig ist hier nur der bereits im Titel des Aufsatzes kenntlich 
gemachte Zusammenhang: » >Going monoclonalk: art, science, and magic in the day-to-day use of hybridoma technology." (Cambrosio/Keating 1988) Cambrosio und Keating greifen in ihrem Aufsatz die Frage auf, welches Verhältnis in wissenschaftlicher Arbeit, im Alltag des Labors, explizites, implizites, stillschweigendes und unsagbares Wissen haben. Wichtig ist vor allem ihre Unterscheidung zwischen stillschweigendem Wissen (tacit knowledge), über das sich prinzipiell reden lässt, und unsagbarem Wissen, über das sich nur unter Drohung von Sanktionen sprechen lässt, weil es herrschenden wissenschaftlichen Gepflogenheiten widerspricht. Diese zweite Kategorie des Wissens thematisieren sie mit dem Begriff der »Kunst« oder der »Magie«. Ihr Argument in Kürze: Im wissenschaftlichen wie auch im außerwissenschaftlichen Alltag bleibt vieles Wissen ungesagt oder wird stillschweigend vorausgesetzt - Wissen ist stumm, es erscheint im Vollzug, es lässt sich teilnehmend erschließen, aber selten abfragen.

Dieses Schweigen muss dabei seinen Grund nicht darin haben, dass sich dieses Wissen nicht oder nur unter Schwierigkeiten verbalisieren ließe, wie es in der klassischen Definition des »tacit knowledge « bei Polanyi angenommen wird (Polanyi 1985). Sondern es könne auch sein, dass dieses Wissen als so trivial angesehen werde, dass es sich nicht lohne, überhaupt darüber zu sprechen oder - und dies ist der interessantere Fall - dass das Wissen den herrschenden Paradigmen widerspreche bzw. man Angst davor habe, sich lächerlich zu machen, oder mit stillschweigenden Denkverboten konfrontiert sei: "much that is unsaid clearly has the possibility of being verbalized but remains, for many reasons, unsayable, unthinkable, trivial, secret, or censored «. Keating und Cambrosio stellen jedoch die These auf, dass dieses Ungesagte oder das Unsägliche durchaus ein wichtiges Element der wissenschaftlichen Praxis sei - es sei Gegenstand von Verhandlung, Diskussion, und (Re-) Konstruktion - jedoch geschehe dies nicht in Workshops oder anderer »offizieller« Wissenschaftskommunikation. (Cambrosio/ Keating 1988: 246)

Keating und Cambrosio beginnen ihre Argumentation mit dem Hinweis, dass die von ihnen untersuchte Technik Mitte der 1980er Jahre von denen, die sie erfolgreich anwandten, als »Kunst« bezeichnet wurde: So werde etwa in technischen Anleitungen darauf hingewiesen, dass Neulinge in diesem Feld die Technik in einem Labor erlernen sollten, das schon langjährige Erfahrung damit gemacht hat; so zitieren sie aus einem Handbuch: 
»It has been a frequent observation that newcomers to the technique are relatively unsuccessful initially and [are successful after some time], although an experienced observer cannot see any difference between the technique used on the first day and in subsequent, successful experiments. « (Cambrosio/Keating 1988: 248) Expertise wird hier nicht etwa damit gleichgesetzt, immer erfolgreich zu sein, sondern mit der Fähigkeit, sich »sicher« in einer experimentellen Situation zurechtzufinden, die durch ein hohes Maß an Unsicherheit und auch Noch-nicht-Wissen charakterisiert sind. So zitieren sie einen in diesem Sinne zum erfolgreichen Experten ausgebildeten Wissenschaftler, mit dem sie ein Interview führten und in dem er seine Lernerfahrung schilderte, wie folgt:

»I consider that the actual [technique ...] has a lot of voodoo. There is a lot of things people do, they don't know why. I don't know why but I just copy what they do and they say: >if you do it differently, it will not work.< They told me I had to spin the [...] cells with the top [of the centrifuge] open. Why the top open? It doesn't make any difference, this is a small, desktop centrifuge, it doesn't matter whether the top is open or closed. I think the history of it is [laughter] that you can't regulate the speed that well, so initially when people used to do it, they would open the top and see how fast it would spin. Now people know how to regulate the speed and they don't really have to look at it anymore, but they leave the top open! They told me I had to leave the top open, I am supposed to be a scientist, I don't believe the top has to be open, but I am not going to put it down, because if the fusion did not work, they would tell me it's because I left the top down«. (Cambrosio/Keating 1988: 253; kursiv SB)

Die zentrale Passage aus meiner Sicht ist hier: I am supposed to be a scientist - meint: ich sollte mich nach streng rationalen Kriterien richten, unter denen die Frage der Kausalität, des objektiven Zusammenhangs von Ursache und Wirkung, die zentrale handlungsleitende Maxime darstellt. Irgendwie gearteter Glaube, der nicht rational zu begründen ist, oder Tradition im Sinne eines >das haben wir immer schon so gemacht , darf bei wissenschaftlichem Handeln eigentlich keine Rolle spielen. So sieht es auch die Wissenschaftsforschung seit Merton oder Luhmann - die beide auf den generalisierten Zweifel als zentralem Merkmal aller modernen Wissenschaft verwiesen hatten. Und dies' entspricht auch dem expliziten Selbstverständnis der meisten Wissenschaftler selbst. Trotzdem hält der In- 
terviewpartner sich an eine - aus seiner Sicht eigentlich unsinnige - Konvention, weil er unfruchtbaren Diskussionen mit den Experten aus dem Weg gehen will. Begründeter Zweifel wird hier sozial zum Schweigen gebracht. Allerdings wäre zu erwarten, dass alle Beteiligten die gewohnte Praxis nach allen Regeln der wissenschaftlichen Kunst in Frage stellten, sobald der Verdacht bestünde, dass sie die Ergebnisse der Experimente verfälschte oder sonst ungewünschte Effekte hätte.

Dass wissenschaftliche Praktiken im Labor oft von den in der Wissenschaftsforschung beschriebenen idealtypischen Verfahrensweisen und rationalem Handeln abweichen, mit teils gravierenden, auch rechtlichen Folgen (vgl. Hughes 2009), wird auch in einem Artikel der beiden Wissenschaftsforscher Kathleen Jordan und Michael Lynch herausgearbeitet. Sie zitieren einen Laborforscher wie folgt:

"I don't think I've ever done exactly the same thing and had it work. I think a lot of times I've changed some variables. ... I mean, the real scientific way to do it would be to change one variable at a time and then try it again, and then keep changing variables and do positive controls and negative controls and all that stuff, and then eventually get it to work. But when you really need it to work you might change a lot of variables at once $[\ldots]$. And then sometimes it works. I can't think $[\ldots]$ you know it is in a way ... in a way it's, you know, magic. But also there are a lot of things you sort of make up your mind as to what could be going wrong, and I don't know if they are ever true, but sometimes when you change things, thinking they are true, then it will work, and so in your mind from then on it's true. You know what I mean?«(Jordan/Lynch 1998: 784f.)

Was er meint? Er erklärt hier, wie es passieren kann, dass man falsche Modelle von Wirkzusammenhängen bildet und, diese nicht in Frage stellt, weil die eigentlich falschen Modelle trotzdem zum Ziel führen. Der Begriff »Magie« wird hier benutzt für instrumentelles Handeln, das auf eigentlich nicht gerechtfertigtem Glauben beruht, das aber erfolgreich ist.

Sowohl im Artikel von Cambrosio und Keating als auch in dem Papier von Jordan und Lynch wird die für wissenschaftliches Handeln zentrale Vorannahme seiner durchgängigen Rationalität in Frage gestellt. Wissenschaftliches Handeln ist demnach nicht immer rational, folgt nicht immer der Prämisse des "generalisierten Zweifels«, das in wissenschaftlichen Praktiken erzeugte Wissen ist nicht immer explizit und auch nicht ohne 
weiteres »generalisiert«, sondern durchaus gebunden an lokale Bedingungen und soziale Arrangements - eben an »Akteur-Netzwerke« im Sinne der Akteur-Netzwerk Theorie. Und vor allem integrieren Wissenspraktiken sehr unterschiedliche und bisweilen sogar gegensätzliche Wissensformen. Um nochmals Cambrosio und Keating zu zitieren:

»We have seen that in the establishment and diffusion of a scientific technique, which may be conceived of as an embedded system of practices, scientists have recourse to many forms of knowledge. That part which may be considered tacit or local depends upon the network of relations within which the scientists work. This network is comprised of a system of heterogeneous elements (theories, machines, patents, products). The articulation of these diverse elements occasions the emergence of the scientists' categories of knowledge. [...] while scientists often present ideal, algorithmic accounts of their work, they also recognize and work with tacit or local dimensions of knowledge whether they be classified as "art « or »magic."In many respects, the scientists' own descriptions of the kinds of knowledge with which they deal on a daily basis are both more precise and more comprehensive than the descriptions offered by sociologists. «(Cambrosio/Keating 1988: 258)

\section{Wissenschaft, Rationalität UND MAgie ALS DAUERTHEMA DER ANTHROPOlogie}

Aus Sicht der Anthropologie oder Ethnologie bietet sich hier ein interessanter Anknüpfungspunkt, denn die Frage, wie sich Magie und Wissenschaft unterscheiden ließen, strukturierte schon im 19. Jahrhundert die leitenden Fragestellungen der Disziplin, die Art der Klassifikation der beschriebenen kulturellen Phänomene und ermöglichte vor allem eine vergleichende Sicht auf kulturelle Praktiken, die entweder - falls sie den Rationalitätskriterien westlicher Wissenschaft folgten - als »modern« und »zivilisiert« oder aber im abweichenden Falle als "vormodern« und »wild« klassifiziert wurden. Der in Ceylon geborene und an der Harvard Universität lehrende Anthropologe Stanley J. Tambiah hat in seinem großartigen Buch »Magic, science, religion, and the scope of rationality« (Tambiah 1990) die fachgeschichtliche Bedeutung der Unterscheidung zwischen Wissenschaft, Magie und Religion herausgearbeitet, die sich - in verschiedenen Spielarten und Akzentsetzungen - bei allen anthropologischen Klassikern 
findet. Aber diese Unterscheidung findet sich auch bei den frühen Soziologen wie Weber oder Durkheim, der Religionswissenschaft oder der frühen Psychologie Wilhelm Wundts und später auch der Freudschen Psychoanalyse; diese Unterscheidung ist zudem eng an ein evolutionistisches Konzept der Entwicklung des menschlichen Denkens und der menschlichen Kultur gebunden: Die Menschheit steigt gleichsam aus den Niederungen magischen, irrationalen Denkens, sie lässt schrittweise animistische Modelle der Welt hinter sich zurück und schreitet auf eine rationalere, wissenschaftliche Weltsicht zu und gelangt schließlich zu einem rationalen Umgang mit der Welt und den Dingen in ihr.

Dies stellt nicht nur die große Erzählung der Moderne dar - und die Selbsterklärung des modernen Menschen -, sondern diese Erzählung begründet das Projekt einer komparativen Sicht auf epistemologische, soziale und kulturelle Phänomene. In Tambiahs Worten:

»How do we understand and represent the modes of thought and action of other societies, other cultures? [The comparative study of cultures] raises not only the question of the mentality of us and other peoples, but also ultimately the issue of rationality itself, and the limits of western >scientism « as a paradigm.« (Tambiah 1990: 3)

Tambiah nimmt seinen Ausgangspunkt bei Sir Edward Tylor, dem Begründer der Anthropologie im viktorianischen Großbritannien, der von 1832 bis 1917 lebte und ab 1884 das Fach in Oxford unterrichtete. Tylor sah die Anthropologie als Modernisierungskraft, die »die Überbleibsel alter, primitiver Kulturen, die auf die Ebene einfachen Aberglaubens herabgesunken seien, zu identifizieren und der Zerstörung anheimzugeben habe«. (ebd.: 44) $\mathrm{Zu}$ diesen abgesunkenen »Resten « vergangener, barbarischer Kulturen zählten vor allem die magischen Künste und die »okkulten Wissenschaften«. Für Tylor gründete magisches Denken in einer universellen menschlichen Fähigkeit zur »Assoziation von Ideen«. Der Fehler magischer Praktiken sei jedoch, »ideale Verbindungen für reale Verbindungen« zwischen Dingen und Gegenstandsbereichen zu halten. Eins seiner vielen Beispiele: Der Wilde bemerke, dass der Hahn krähe, wenn die Sonne aufgehe. Daraus schließe er nun, dass die Sonne aufgehen werde, wenn man den Hahn zum Krähen bringe. Es sind diese gleichsam >verdrehten〈 Kausalitätsannahmen, die für Tylor magische Praktiken aber auch religiöse Überzeugungen zur Antithese wissenschaftlichen Handelns und aufgeklärten Denkens machen. 
Im Gegensatz dazu sah Émile Durkheim hier eher Kontinuitäten: für ihn stellen elementare religiöse Ideen Vorläufer der späteren Wissenschaft dar. Tylor dagegen vertrat stets die klare These, dass die in Magie und Religion vorherrschende Denkweise dem wissenschaftlichen Denken diametral entgegengesetzt sei.

An dieser Position, die für die frühe Anthropologie und Ethnologie auch international prägend wurde und mit der ein Konzept verankert wurde, das sich als »kognitiver Evolutionismus« bezeichnen lässt, wurde in England vor allem durch die Anthropologen Edward E. Evans-Pritchard und Bronislaw Malinowski grundsätzliche Kritik geübt. Evans-Pritchard kritisierte vor allem die psychologisierende Sicht von Tylor: »The error here was in not recognizing that the [false] associations [between domains] are social and not psychological stereotypes, and that they occur therefore only when evoked in specific ritual situations, which are also of limited duration.« (zit. n. Tambiah 1990: 51) So betonte Evans-Pritchard, dass Personen in ihrem Denken und Handeln zwischen mythischen und alltäglich-praktischen Kontexten wechseln könnten - es sich beim magischen Denken und Handeln also um situationsabhängige, sozial erzeugte Modi des Denkens und Argumentierens handele. So habe etwa ein Nuer-Dorf heilige Pfosten, die die Ahnen symbolisierten - Objekte, die zusammen mit ihrer unmittelbaren Umgebung zu besonderen Zeiten heilig seien, weil die Geister der Ahnen in ihnen anwesend seien. Aber außerhalb dieser speziellen Ritualzeiten würden die gleichen Gegenstände alläglich und unmagisch behandelt. (Tambiah 1990: 92)

Damit markiert Evans-Pritchard eine grundsätzlich andere Position als Tylor: Für Tylor war der »Wilde« vollständig in einem magischen Weltbild gefangen; ebenso argumentierte Lévy-Bruhl in seinen frühen Schriften (Lévy-Bruhl 1959). Der »Wilde« habe gleichsam eine kindliche Psyche; für Evans-Pritchard hingegen unterschied den Wilden vom Zivilisierten lediglich, dass ersterer in bestimmten, sozial wohl definierten Zusammenhängen zu magischen Interpretationen von Geschehnissen neigte. (Vgl. hierzu ähnlich auch Malinowski 1992 (1948): 25) In seinem grundlegenden Buch »Hexerei, Orakel und Magie bei den Zande« schreibt Evans-Pritchard etwa:

»Im Zandeland stürzt manchmal ein alter Getreidespeicher ein. Daran ist nichts Bemerkenswertes. Jeder Zande weiß, dass Termiten die Stützbalken im Laufe der Zeit zernagen und daß auch das härteste Holz nach Jahren der Beanspruchung verrottet. 
Nun ist aber ein Speicher immer zugleich das Sommerhaus eines Zande Gehöfts und die Leute sitzen während der Mittagshitze darunter, plaudern oder spielen das afrikanische Lochspiel oder sind mit irgendeiner handwerklichen Tätigkeit beschäftigt. Infolgedessen kann es passieren, daß gerade dann, wenn er einstürzt, Leute daruntersitzen und verletzt werden, denn es ist ein massiver Bau aus Balken und Lehm [...]. Warum mußten gerade im Moment des Einsturzes ausgerechnet diese Leute unter dem betreffenden Speicher sitzen? Daß er einstürzen mußte, ist leicht verständlich. Aber warum mußte er gerade in dem Moment, als ausgerechnet diese Leute daruntersaßen, einstürzen? Er hätte schon seit Jahren einstürzen können, warum also tat er es gerade dann, als bestimmte Leute seinen behaglichen Schutz suchten? Wir sagen, daß der Speicher einstürzte, weil seine Stützen von Termiten weggefressen wurden: das ist die Ursache, die den Einsturz des Speichers erklärt. Wir sagen auch, daß Leute gerade darunter saßen, weil es die heißeste Zeit des Tages war und sie dachten, daß es ein bequemer Ort zum Reden und Arbeiten sein würde: das ist die Ursache dafür, daß zum Zeitpunkt seines Einsturzes Leute unter dem Speicher waren. Für uns besteht der einzige Zusammenhang zwischen diesen beiden unabhängig voneinander verursachten Sachverhalten in der Koinzidenz von Zeit und Ort. Wir haben keine Erklärung dafür, warum die beiden Kausalketten sich zu einem bestimmten Zeitpunkt und an einem bestimmten Ort überschnitten, da es keine Interdependenz zwischen ihnen gibt. Die Philosophie der Zande kann dazu das fehlende Glied liefern. Ein Zande weiß, daß die Stützen von Termiten unterhöhlt waren und daß Leute unter dem Speicher saßen, um der Hitze und dem gleißenden Sonnenlicht zu entgehen. Aber er weiß außerdem, warum diese beiden Ereignisse zur genau gleichen Zeit am gleichen Ort eintraten. Es war eine Folge der Wirkung von Hexerei.« (EvansPritchard 1978: 65f.)

Evans-Pritchard weist damit darauf hin, dass magisches Denken Erklärungen zur Verfügung stellt, wo im wissenschaftlichen Weltbild eine Leerstelle bleibt: allein »unerklärbarer Zufall« sei die in der Moderne zur Verfügung stehende Nicht-Erklärung, warum bestimmte Dinge geschehen, die außerhalb erklärbarer Kausalitätsverhältnisse stehen. Das Wissen um Hexerei ermögliche dagegen den Zande, solche kontingenten Ereignisse zu verstehen und sozial zu bearbeiten - etwa durch Gegenzauber oder Bestrafung der Schuldigen.

Ganz in dieser Linie argumentiert auch Bronislaw Malinowski, der »Erfinder« der anthropologischen Feldforschung - und damit derjenige, der 
sich von Tylors »spekulativem Idealismus« scharf abgrenzt. ${ }^{2}$ Auch Malinowski unterschied klar zwischen Magie und Wissenschaft, wobei er Wissenschaft als eine >profane` Aktivität verstand, während Magie und Religion als >heilige Aktivitäten anzusehen seien. Für Malinowski hatten jedoch beide Bereiche universelle Bedeutung in allen Kulturen. Seine zentrale Frage: Hat der Wilde ein rationales Verhältnis zur Welt, meistert er seine Umgebung mit rationalen Mitteln? Und weiter: Kann dieses primitive Wissen als eine rudimentäre Form der Wissenschaft angesehen werden? Beide Fragen werden von ihm grundsätzlich bejaht, wobei er seine empirischen Beispiele aus seinen Feldforschungen bei den Trobriandern gewinnt, einem indonesischen Inselvolk, bei dem er während des Ersten Weltkrieges - teilweise unfreiwillig - festsaß und an ihrem Alltag forschend teilnahm.

So schreibt er etwa, dass der sehr erfolgreiche Gartenbau, den die Trobriander betreiben, von ihrem umfangreichen Wissen über unterschiedliche Bodenqualitäten, über die Eigenschaften unterschiedlicher Nutzpflanzen, die wechselseitige Adaption beider Faktoren und nicht zuletzt von ihrem Wissen um die Wichtigkeit harter und akkurater Arbeit abhingen. In ihrer Arbeit in den Gärten seien sie stets durch ein klares Wissen um Wettereinflüsse und Jahreszeiten, Pflanzen und Schädlinge, und Bodenverhältnisse geleitet - und durch die Überzeugung, dass ihr Wissen wahr und verlässlich sei, dass man auf es rechnen könne und das Handeln danach minutiös ausgerichtet werden müsse. Doch in all dieses rationale Handeln sei Magie eingewoben, eine Serie von rituellen Praktiken, die während des ganzen Jahres in den Gärten in genau eingehaltener Reihenfolge durchgeführt würden. Ein oberflächlicher Beobachter - so Malinowski - könne den Eindruck gewinnen, dass rationale und magische Handlungen von den Eingeborenen nicht unterschieden würden. Dies sei jedoch nicht der Fall - zwar werde Magie von den Trobriandern als unverzichtbar erachtet für das Gedeihen

2 Zum Einfluss der Völkerpsychologie Wilhelm Wundts (Wundt, Wilhelm Maximilian: Völkerpsychologie. Eine Untersuchung der Entwicklungsgesetze von Sprache, Mythus und Sitte. Bd. 1: Die Sprache, Teil 1. Leipzig: 1900, Wilhelm Engelmann.) und der frühen Wissenschaftsphilosophie u.a. Ernst Machs (Mach, Ernst: Erkenntnis und Irrtum. Skizzen zur Psychologie der Forschung. 1905, J. A. Barth.), der ebenfalls von einer Kontinuität wissenschaftlicher und alltäglicher Wissenspraktiken ausging, auf Malinowskis theoretische Sicht vgl. (Tambiah 1990: 65). 
der Gärten, aber dies hieße nicht, dass sie alle Erfolge der Magie zurechneten. Ich zitiere in Übersetzung:

»Wenn Sie einem Eingeborenen empfehlen wollten, dass er doch seinen Garten allein mit Magie bestellen und seine Arbeit einstellen solle, dann würde er lediglich über Ihre Einfalt lächeln. Er weiß genauso gut wie Sie, dass es natürliche Bedingungen und Ursachen gibt, und durch seine Beobachtungen weiß er, dass er diese natürlichen Kräfte durch mentale und physische Arbeit kontrollieren kann. [...] Wenn die Zäune zusammengefallen sind, wenn der Samen zerstört ist oder weggewaschen wurde, dann wird er seine Zuflucht nicht in der Magie suchen, sondern in der Arbeit, angeleitet durch Wissen und Vernunft. Aber seine Erfahrung hat ihn auch gelehrt, dass trotz all seiner Voraussicht und seiner Anstrengungen Kräfte existieren, die ihm in einem Jahr Überfluss und Fruchtbarkeit gewähren [...] während in einem anderen Jahr dieselben Kräfte Pech und Unglück bringen, ihn von Anfang bis Ende verfolgen und all sein Bemühen und sein bestes Wissen fruchtlos machen. Um diese Kräfte zu kontrollieren - und nur diese - setzt er Magie ein.« (Malinowski 1992 (1948): 28f.)

Für Malinowski besteht also eine klare Trennung zwischen einerseits einem Wirklichkeitsbereich, in dem gut verstandene Bedingungen, in dem ein klares Verständnis von kausalen Beziehungen herrschen, in dem normales Wachstum oder gewöhnliche Schädlinge anzutreffen sind, Faktoren, denen mit einfachen Mitteln begegnet werden kann. Andererseits, gibt es einen Bereich der Wirklichkeit, der sich solch positivem Wissen entzieht und in dem alltägliches Handeln keine Wirkung hat. Gegen diese Probleme wird Magie eingesetzt.

Malinowski erläutert dies noch an einem weiteren Beispiel ausführlich: Am Fischfang und dem Kanubau der Trobriander. Er gesteht ihnen ein ausgezeichnetes Erfahrungswissen im Bootsbau zu, sie verfügten über empirisches Wissen der verwendeten Materialien, der einzusetzenden Technologien und der Hydrodynamik. Sie verfügten über ein ganzes System von Prinzipien für die Seefahrt, das in einer reichen und komplexen Terminologie festgehalten sei und auf deren Einhaltung sie peinlich achteten. Doch sei ein bedeutender Unterschied festzustellen zwischen dem Fischfang in den Lagunen und auf hoher See: Während in den an den inneren Lagunen der Inseln liegenden Dörfern Fischfang auf einfache und höchst verlässliche Weise durch »poisoning« geschehe und stets große Erträge erziele, ohne dass die Fischer Gefahren auf sich nehmen müssten oder Ungewisshei- 
ten zu begegnen hätten, würden in den Dörfern, die direkt am Ufer des Ozeans lägen, gefährliche Arten des Fischfangs praktiziert. Hier seien die Erfolgsaussichten ungewiss und die Erträge schwankten beträchtlich. Während bei der Lagunenfischerei, wo die Fischer sich vollständig auf ihr Wissen und ihre Erfahrung verlassen könnten, keinerlei Magie praktiziert werde, würden bei den Hochseefischern ausgiebig magische Rituale praktiziert, um gute Resultate und sichere Heimkehr zu garantieren.

Diese Befunde generalisiert Malinowski; für ihn sind Wissenschaft und Magie zwei unabhängige, doch aufeinander bezogene Wissenssysteme, die in unterschiedliche soziale Praktiken eingebettet sind und die auf sehr unterschiedliche Problemkonstellationen bezogen sind. Nochmals Malinowski:

»Science $[\ldots]$ is based on the normal universal experience of everyday life, experience won in man's struggle with nature for his subsistence, and safety, founded on observation, fixed by reason. ${ }^{3}$ Magic is based on specific experience of emotional states in which man observes not nature but himself [...]. Science is founded on the conviction that experience, effort, and reason are valid; magic on the belief that hope cannot fail nor desire deceive. The theories of knowledge are dictated by logic, those of magic by the association of ideas under the influence of desire. As a matter of empirical fact, the body of rational knowledge and the body of magical lore are incorporated each in a different tradition, in a different social setting and in a different type of activity, and all these differences are clearly recognized by the savages." (Malinowski 1992 (1948): 87)

Um nochmals auf die Schilderung des Laborwissenschaftlers zurückzukommen, der aufgefordert wurde, den Deckel der Zentrifuge offen zu lassen, damit sich beim Experiment der Erfolg einstellt: auch hier geht es um kausal nicht vollständig verstandene Zusammenhänge, die es auf jeden Fall als »sicherer« erscheinen lassen, von einem bestimmten Arrangement nicht abzuweichen, weil es sich einmal als erfolgreich erwiesen hat. Aber was der Labormitarbeiter als "voodoo« oder magisch bezeichnet, ist Teil des

3 Interessant sind hier auch die Querbezüge und Ähnlichkeiten zur Perspektive der amerikanischen Pragmatisten William James und John Dewey (Dewey, John: How we think. Boston: 1910, D.C. Heath \& Co.) - eine Parallele, die auch Tambiah in seiner Darstellung erwähnt, aber nicht vollständig verfolgt. 
gleichen sozialen Settings, eines einheitlichen Aktivitäts-Typs und der gleichen - in Kochrezepten festgehaltenen und mündlich wie praktisch weiter gegebenen - »Tradition«. Und ob die Eingeborenen der Labore den Unterschied zwischen beiden Wissenstypen so präzise zu unterscheiden wissen, wie dies Malinowski bei den Eingeborenen der Trobriand-Inseln unterstellt, ist - folgt man der Schilderung des Laborwissenschaftlers zumindest fraglich. Es wäre zumindest eine Frage wert, ob die Selbstreflexion hinsichtlich der in Aktion gesetzten Wissenstypen im Alltag oder in der Wissenschaft stärker ausgeprägt ist.

Festzuhalten ist: man muss nicht gleich annehmen, dass weder Eingeborene noch zivilisierte Menschen keinen Unterschied machen zwischen dem Magischen und dem Nicht-Magischen, wie dies der britische Anthropologe Edmund R. Leach in einer Kritik der Malinowski'schen Perspektive konstatierte:

»Malinowski maintained, no doubt rightly, that Trobrianders are at least as rational as twentieth-century Europeans. [...] Were he seems to err is in maintaining that the ordinary man distinguishes consistently between the magical and the non-magical. [...] In seeking to break down the dichotomy between savagery and civilization Malinowski argued that primitives were just as capable as Europeans of making such distinctions. [...] He would have had a much better case if he had insisted that Europeans are ordinarily just as incapable as Trobrianders of distinguishing the two categories.«(Leach 1957, zit. n. Nader 1996: 260)

Dies - so scheint mir - ist ein klassischer Fall von »das Kind mit dem Bade ausschütten«: Der richtige Hinweis darauf, dass magische Praktiken ein durchaus alltägliches Phänomen in der westlich-rationalen Moderne sind ebenso wie rationales Handeln bei Menschen außerhalb dieser Moderne, setzt nicht notwendigerweise eine Differenzierungsunfähigkeit gegenüber diesem Unterschied voraus - lediglich die Schwierigkeit, in einem Kontinuum stets klare Wesensunterschiede festzumachen und im Alltag diese Unterscheidungsfähigkeit konsistent anzuwenden. Malinowski selbst war sich dem nur zu bewusst: »I was not able to judge for myself where rational procedure ended and which were the supererogatory activities, whether magical or aesthetic.« (Malinowski (1935) zit. n.Leach 1957: 128) Doch hierbei handelt es sich um empirisches, nicht um ein prinzipielles Problem. 
Aber nicht nur für die britische Sozialanthropologie gilt, dass sie sich bereits frühzeitig mit der Frage der Rationalität und dem Unterschied zwischen wissenschaftlichem und praktischem Umgang mit der Realität auseinandersetzte. Auch die Europäische Ethnologie griff diese Thematik auf - anders als die Ethnologie jedoch am Phänomen einer zunehmenden Technisierung des Alltagslebens. Die Rationalitätsproblematik kam hier gleichsam indirekt ins Spiel, da Technik als Anwendung wissenschaftlicher Rationalität und als mächtiger Rationalisierungsfaktor verstanden wurde. Bezeichnenderweise ist es diese in den 1960er Jahren aufkommende Debatte, die den Übergang von einer romantisierenden Volkskunde zu einer ethnographisch orientierten, sozialwissenschaftlich und historisch argumentierenden Europäischen Ethnologie markiert.

\section{HERMANN BAUSinger: VOLKSKULTUR IN DER TECHNISCHEN WELT}

Der Tübinger Germanist und Volkskundler Hermann Bausinger veröffen tlichte seine Habilitationsschrift 1961 unter dem Titel »Volkskultur in der technischen Welt«. Erklärtes Ziel seiner Analysen - so schreibt er - sei es, neue, der »technischen Welt« angemessene und die einzelnen Fachgebiete der Volkskunde übergreifende Kategorien zu bilden. Hiermit möchte er einem von ihm wahrgenommenen zentralen Defizit der Nachkriegsvolkskunde begegnen, die - statt neue Begriffe und Kategorien zu bilden - »neue Gegenstände und Bevölkerungsgruppen an den alten Begriffen« (Bausinger 1986: 14) gemessen habe. Mit dieser Programmatik wird nun aber nichts weniger als ein »paradigmatischer« Sprung in der Nachkriegsvolkskunde eingeklagt und das Forschungsterrain neu vermessen: weg von der konservierenden Reliktforschung, hin zur Analyse gegenwärtiger Volkskultur, verstanden als die »reale Welt der kleinen Leute«. (ebd.: 9) Mit seiner Studie leitete Bausinger die Abkehr von der alten Volkskunde und deren Neuerfindung als empirische Kulturwissenschaft oder Europäische Ethnologie ein. Sein Ansatzpunkt ist dabei die Kritik der zentralen Vorannahme zahlreicher zeitgenössischer volkskundlicher Arbeiten: nämlich dass zwischen Volkswelt und technischer Welt ein unüberbrückbarer Gegensatz bestünde. Bausinger macht bereits in der Überschrift des ersten Kapitels klar, dass dieser Gegensatz unhaltbar sei; stattdessen - 
so die Überschrift - sei »Die technische Welt als `natürlicheく Lebenswelt» anzusehen.

Ausgangspunkt des ersten Kapitels der Studie ist eine wenig schmeichelhafte Standortbestimmung der Volkskunde zu Beginn der 1960er Jahre: Danach habe die bereits von Wilhelm Grimm entwickelte und von der Volkskunde weitgehend unbefragt vom 19. Jahrhundert bis in die Gegenwart mitgeführte Vorstellung, dass sich Volkswelt und »technische Welt« in unversöhnlichem Gegensatz gegenüberstünden, zu einer Abgrenzung der Forschungsfelder geführt, die volkskundliche Forschungsarbeit auf Reliktzonen verweise und das Fach mit der »Ausbreitung der Technik in immer engere Winkel und - was schlimmer ist - in eine immer zwielichtigere Haltung zur Wirklichkeit« (ebd.: 19) dränge. Das Bild, das Bausinger hier weitgehend unter Verzicht auf drastische Worte zeichnet, lässt sich pointiert zusammenfassen: Der Wissenschaft, die sich ausschließlich mit immer marginaler werdenden Marginalitäten beschäftigt, droht letztlich selbst die Marginalisierung, die Substanzgefährdung der Untersuchungssujets schlägt um in disziplinäre Bestandsgefährdung. Was mit diesen Überlegungen auf die Tagesordnung der Fachdiskussion gesetzt wird, geht damit deutlich über eine Ausweitung der Forschungsthemen hinaus: es geht um die theoretische und methodische Neu-Ausrichtung der Disziplin.

Aus dieser geschilderten Krise führt für Bausinger nur eine grundlegende Umorientierung heraus, die bei den Vorannahmen ansetzen muss, die das Fach und seine Arbeitshypothesen konstituieren. Zum Zweck dieser wortwörtlich radikalen Kritik rekonstruiert er vier Antinomien, die den in volkskundlichen Arbeiten verbreiteten Befund eines Gegensatzes zwischen Volkskultur und »technischer Welt« stützten: So werde

- die Volkswelt als ungeschichtlich, die »technische Welt« dagegen als in höchstem Maße geschichtlich konzipiert,

- die »beseelte Volkswelt« würde dem »mechanischen Charakter der technischen Welt« gegenübergestellt,

- der von organischen Gemeinschaften bestimmten Volkskultur werde die Vorstellung der organisierten Gesellschaft entgegengesetzt und schließlich werde

- die Opposition Irrationalität-Rationalität zur Unterscheidung zweier grundsätzlich anderer Phänomenbereiche benutzt: »in der technischen 
Welt herrschte demnach die rationale Konstruktion, während die Volkswelt ihre stärksten Impulse aus nichtrationalen Tiefen erhielte.« (ebd.: 20)

Bausinger analysiert diese das Konzept »Volkskultur« bestimmenden Gegensatzpaare als Bestandteile eines antimodernen, weitgehend in der Romantik entstandenen Gegenbildes zur Industriegesellschaft. Durch Umkehrung dieser Oppositionen oder durch deren Relativierung versucht er dieses Konzept als unhaltbar zu widerlegen und setzt diesen Dichotomien seine These der »Natürlichkeit des Technischen« entgegen. An zahlreichen empirischen Befunden belegt er, dass nach einer kurzen Phase, in der Technik als Bedrohung erfahren wird, sie schnell als selbstverständlicher Teil in die Alltagskultur integriert wird und über Volkslieder, Reime, Erzählungen, oder Witze in alltägliche Vorstellungswelten eingebaut wird. Technik steht demzufolge nicht länger in unversöhnlichem Gegensatz zur Volkskultur, sondern wird einer ihrer bestimmenden Faktoren, womit die Volkskultur auch und gerade in der »technischen Welt« zu einem volkskundlichen Forschungsfeld wird. Bausinger greift hiermit Thematiken auf, die Jahre später in techniksoziologischen und technikhistorischen Untersuchungen systematisch analysiert wurden. (SOzIALE Konstruktion von TeChNOLOGIE und AKTEUR-NETZWERK THEORIE)

Der Vektor der Argumentation ist damit auf eine Revision des Volkskulturkonzeptes der Volkskunde gerichtet; Technik selbst wird hierbei jedoch nur am Rande zum Thema und bleibt begrifflich weitgehend un(ter)bestimmt. Als zusammenfassende Definition ergibt sich aus dem Text, dass Technik vor allem angewandte Naturwissenschaft ist, die im Binnenraum durch eine rationale Struktur gekennzeichnet ist; eine Rationalität, die jedoch die »technische Welt« bisweilen auf gefährliche Art vermissen ließe. So sei die Benutzung der Technik oft nicht »bewusst« im eigentlichen Sinne, sondern oft »naives Tun« (ebd.: 21), das sich nicht mit der Funktion der Apparaturen auseinandersetze. Aus der Beobachtung, dass Maschinen für ihre Nutzer vorübergehend eine Erweiterung des Körperschemas bedingen und somit nicht mehr der Umwelt zugerechnet werden können, schließt Bausinger, »daß Erscheinungen der Technik durchaus nicht immun oder auch nur spröde sind gegen vorrationale Verhaltens- und Denkformen«. (ebd.: 21) Besonders deutlich zeige sich dies an zu beobachtenden » >magischen` Haltungen und Reaktionen« gegenüber der Technik, die bisweilen 
beim Versagen von Apparaturen aufträten; insbesondere beim Auftreten von Defekten zeige sich so, dass die »Natürlichkeit« des Technischen nicht mit Beherrschung, sondern meist nur - stets gefährdetes - Ergebnis des Umgangs und der Gewöhnung sei. Diese Reaktionen dürften jedoch nicht, wie vielfach geschehen, als Relikte volkstümlichen Denkens interpretiert werden, da es sich hierbei um »Regressionen, von der Technik selber ausgelöste Rückgriffe auf genetisch frühere Stufen $\aleph^{4}$, bzw. um »das genetisch Ältere und damit auf tiefere Schichten des Seelischen « (ebd.: 45) handele. Bausinger legt so nahe, dass es sich beispielweise bei »abergläubischen" Verhaltensweisen um eine durchaus moderne, kompensative Bewegung handelt, mit der die übergroße Komplexität der technischen Welt reduziert werden solle.

Damit werden als künftige Forschungsrichtungen markiert: einerseits das Thema »Natürlichkeit des Technischen« (besser: das Selbstverständlich-Werden), das etwa an volkskundlich vertrauten Sujets wie popularem Erzählen erforscht werden könne, und andererseits das Thema temporärer Regressionen, bei deren Analyse ebenfalls spezifische Kompetenzen der Volkskunde eingebracht werden können. Diese Beschränkung, scheint sich aus dem Versuch Bausingers zu ergeben, sich bei seinem Reformversuch doch weitgehend innerhalb der von der Volkskunde besetzten Grenzen der Forschungsfelder und ihrer Methodik zu bewegen. Bemerkenswert ist weiter, dass der individuelle und kollektive Prozess, in dem Technik angeeignet wird, kaum problematisiert wird. Ebenso wenig in den Blick kommen konnte so die zwischen - als unkompliziert konzipierte - Aneignung und Regression liegende Phase des alltäglichen Umgangs mit funktionierender Technik. Bausinger selbst wies in einer späteren Kritik seines Buches auf ein damit eng verwandtes Problem hin. Die angenommene Verlaufsvorstellung »Bedrohung, Aneignung, Regression« biete zwar erste Ordnungskategorien, doch handele es sich dabei um ein weitgehend mechanisches Konstrukt eines »Stimulus-Response-Modells«, bei dem etwa sozialspezifische Faktoren bei der Auseinandersetzung mit Technik vernachlässigt würden.

4 Bausinger (ebd.: 42) setzt »magisch« hier und an anderen Stellen bewusst in Anführungszeichen, um anzudeuten, daß er diesen Begriff als unscharfen - und nicht exakt wissenschaftlichen - Sammelbegriff verwendet. Hier kann nicht auf das durchaus problematische, dem Sozialevolutionismus entlehnte Konzept der »genetisch früheren Stufen» eingegangen werden, das Bausinger in seinem Buch weitgehend unkritisch übernimmt. 
Außerdem seien die in seinem Buch herangezogenen Beispiele - sowohl für Aneignungen wie Regressionen - meist von einer beträchtlichen »Technikdistanz« gekennzeichnet gewesen. (Bausinger 1981: 232)

Malinowski und Bausinger thematisieren beide magisch oder irrational erscheinende Praktiken und deren Verhältnis zu »rationalem«, wissenschaftlich legitimiertem Handeln. Für beide ist offensichtlich, dass Alltagshandeln nie nur rational oder magisch ist, sondern dass Mischungsverhältnisse und Ambivalenzen in Rechnung zu stellen sind. Damit stellen beide etablierte Auffassungen über die scharfe Trennung zwischen rationalem Handeln und seinem Gegenbild in Frage, halten dabei aber an dem von Max Weber in die Sozialwissenschaft eingeführten Konzept der Rationalität fest - sie ist vor allem realisiert als Zweckrationalität. Trotzdem ist Rationalität bei beiden kein normatives, sondern ein empirisches Problem. Und für beide ist offensichtlich, dass es deshalb systematischer Beobachtung und sorgfältiger, kontextueller Interpretation bedarf, um rationales und weniger rationales Handeln in Aktion analysieren zu können.

\section{LITERATUR}

Bausinger, Hermann (1986): Volkskultur in der technischen Welt, Stuttgart: Kohlhammer.

Bausinger, Hermann (1981): »Technik im Alltag«. Zeitschrift für Volkskunde 2, S. 227-242.

Cambrosio, Alberto/Keating, Peter (1988): »Going Monoclonal: Art, Science, and Magic in the Day-to-Day Use of Hybridoma Technology«. Social Problems, 35(3), S. 244-260.

Dewey, John (1910): How we think, Boston: D.C. Heath \& Co.

Evans-Pritchard, Edward E. (1978): Hexerei, Orakel und Magie bei den Zande. Von Eva Gillies gekürzte und eingeleitete Ausgabe, Frankfurt/M.: Suhrkamp.

Hacking, Ian (1996): Einführung in die Philosophie der Naturwissenschaften, Stuttgart: Reclam.

Hughes, Virginia (2009): »Markers of dispute«. Nature Medicine, 15(12), S. $1339-1343$. 
Jordan, Kathleen/Lynch, Michael (1998): »The Dissemination, Standardization and Routinization of a Molecular Biological Technique «. Social Studies of Science, 28(5/6), S. 773-800.

Kuhn, Thomas S. (1976): Die Struktur wissenschaftlicher Revolutionen, Frankfurt/M.: Suhrkamp.

Leach, Edmund R. (1957): »The epistemological Background to Malinowski’s Empiricism«. In: Raymond Firth (Hg.), Man and Culture, London: Routledge, S. 119-137.

Lévy-Bruhl, Lucien (1959): Die geistige Welt der Primitiven, Düsseldorf [u.a.]: Diederichs.

Mach, Ernst (1905): Erkenntnis und Irrtum. Skizzen zur Psychologie der Forschung, Leipzig: J. A. Barth.

Malinowski, Bronislaw (1948/1992): Magic, Science and Religion and other Essays. With an introduction by Robert Redfield, Prospect Hights: Waveland Press.

Nader, Laura (1996): »The Three-Cornered Constellation. Magic, Science, and Religion Revisited«. In: Laura Nader (Hg.), Naked Science: Anthropological Inquiry into Boundaries, Power, and Knowledge, New York, London: Routledge, S. 259-275.

Polanyi, Michael (1985): Implizites Wissen, Frankfurt/M.: Suhrkamp.

Tambiah, Stanley J. (1990): Magic, Science, Religion, and the Scope of Rationality, Cambridge: Cambridge University Press.

Wundt, Wilhelm M. (1990): Völkerpsychologie. Eine Untersuchung der Entwicklungsgesetze von Sprache, Mythos und Sitte. Bd. 1: Die Sprache, Teil 1., Leipzig: Wilhelm Engelmann. 